\title{
Measurements and tests on FBK silicon sensors with an optimized electronic design for a CTA camera
}

\author{
G. Ambrosi ${ }^{(1)}$, M. Ambrosio ${ }^{(2)}$, C. Aramo ${ }^{(2)}$, E. Bissaldi ${ }^{\dagger(3,4)}$, M. Capasso $^{(5)}$, \\ D. Corti ${ }^{(6)}$, A. de Angelis ${ }^{(6)}$, F. de Palma ${ }^{(7,8)}$, F. Ferrarotto ${ }^{(9)}$, A. Ferri ${ }^{(10)}$, \\ S. Garrappa ${ }^{(5)}$, N. Giglietto ${ }^{(5,8)}$, F. Giordano ${ }^{*}{ }^{(5,8)}$, A. Gola ${ }^{(10)}$, M. Ionica ${ }^{(1)}$, \\ M. Iori ${ }^{(9,11)}$, F. Licciulli ${ }^{(12)}$, M. Mariotti ${ }^{(6)}$, C. Marzocca ${ }^{(8,12)}$, R. Paoletti ${ }^{(13)}$, \\ C. Piemonte ${ }^{(10)}$, V. Postolache ${ }^{(1)}$, R. Rando ${ }^{(6)}$, C. Stella ${ }^{(4,14)}$, P. Vallania ${ }^{(15,16)}$, \\ C. Vigorito ${ }^{(15,17)}$ \\ ${ }^{(1)}$ INFN-Sezione di Perugia; ${ }^{(2)}$ INFN-Sezione di Napoli; ${ }^{(3)}$ Dipartimento di Fisica, Università \\ di Trieste; ${ }^{(4)}$ INFN-Sezione di Trieste-Udine; ${ }^{(5)}$ Dipartimento Interateneo di Fisica, Università \\ e Politecnico di Bari; ${ }^{(6)}$ INFN-Sezione di Padova; ${ }^{(7)}$ Università Telematica Pegaso; ${ }^{(8)}$ INFN- \\ Sezione di Bari; ${ }^{(9)}$ INFN-Sezione di Roma I; ${ }^{(10)}$ FBK-Trento; ${ }^{(11)}$ Sapienza-Università di \\ Roma; ${ }^{(12)}$ Politecnico di Bari; ${ }^{(13)}$ INFN-Sezione di Pisa; ${ }^{(14)}$ Dipartimento di Chimica, Fisica e \\ Ambiente, Università di Udine; ${ }^{(15)}$ INAF-Osservatorio Astrofisico di Torino; ${ }^{(16)}$ INFN-Sezione \\ di Torino; ${ }^{(17)}$ Dipartimento di Fisica, Università di Torino. \\ $\dagger$ E-mail: Elisabetta.Bissaldi@ts.infn.it, Francesco.Giordano@ba.infn.it
}

In October 2013, the Italian Ministry approved the funding of a Research \& Development (R\&D) study, within the "Progetto Premiale TElescopi CHErenkov made in Italy (TECHE)", devoted to the development of a demonstrator for a camera for the Cherenkov Telescope Array (CTA) consortium. The demonstrator consists of a sensor plane based on the Silicon Photomultiplier (SiPM) technology and on an electronics designed for signal sampling. Preliminary tests on a matrix of sensors produced by the Fondazione Bruno Kessler (FBK-Trento, Italy) and on electronic prototypes produced by SITAEL S.p.A. will be presented. In particular, we used different designs of the electronics in order to optimize the output signals in terms of tail cancellation. This is crucial for applications where a high background is expected, as for the CTA experiment.

Science with the New Generation of High Energy Gamma-ray experiments, 10th Workshop - Scineghe2014 04-06 June 2014

Lisbon - Portugal

* Speaker. 


\section{The prototype}

\subsection{The Sensors}

The devices employed for the realization of the matrix prototype make use of the FBK [1] Near Ultra-Violet (NUV) SiPM technology. The basic structure of these SiPMs consists of a $\mathrm{p}^{+}-\mathrm{n}$ junction, whose design is optimized for the detection in the blue-NUV region of the electro-magnetic spectrum [2]. They show low breakdown voltages (around 25.6 V) with a slight temperature dependence of about $24 \mathrm{mV} /{ }^{\circ} \mathrm{C}$. Moreover, these devices also show a Photon Detection Efficiency (PDE) of about $24 \%$ at $380 \mathrm{~nm}$ for $2 \mathrm{~V}$ of over-voltage (OV). In particular, the matrix we tested consists of 16 SiPMs with $50 \mu \mathrm{m}$ cells, for a total equipped area of $12 \times 12 \mathrm{~mm}^{2}$ each (see Figure 1 , left panel).
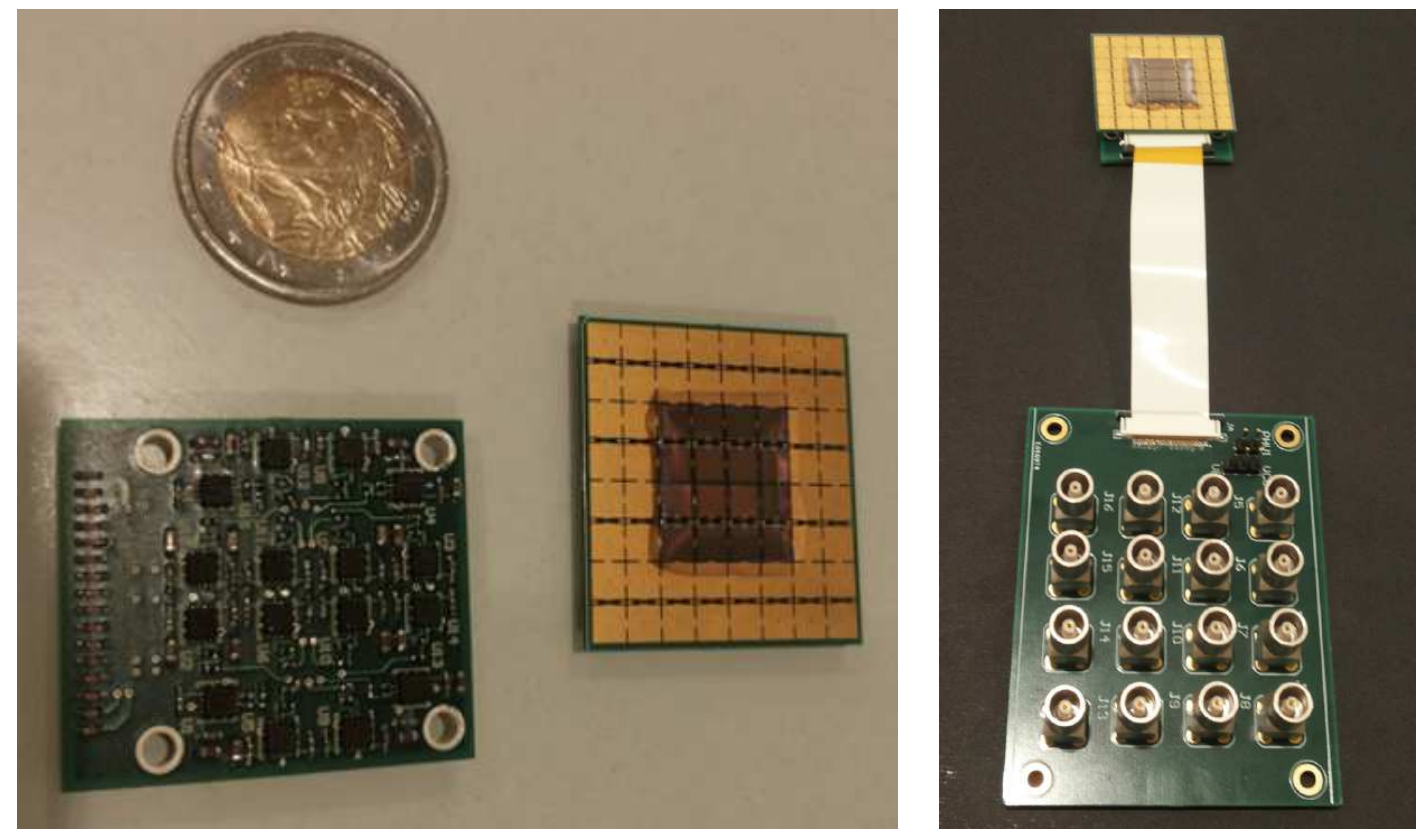

Figure 1: Left panel: On the right, we can see the assembled matrix with the protective layer of epoxy resin. On the left, the printed circuit board (PCB) with the 16 AD8000 OPAs. On the top, Dante's face for size comparison. Right panel: Another PCB showing 16 LEMO connectors for the readout.

\subsection{Front End Electronics}

We designed a preamplifier based on an AD8000 operational amplifier (OPA) in transimpedance configuration. The design consists of $20 \Omega$ decoupling resistances with a $1 \mathrm{k} \Omega$ feedback resistance. The current signal from the SiPM is fed to the preamplifier in DC mode, as illustrated in Figure 2.

The left panel of Figure 3 shows the current signal from the preamplifier corresponding to five photo-electrons (p.e.). The very fast rise time is due to the development of the current signal in the SiPM junction and it is about $100 \mathrm{ps}$ long. Moreover, a fast decay followed by a very long tail is clearly visible. This long tail is due to the recovery time obtained by the product of the quenching resistor times the single cell capacitance value, and it is about $100 \mathrm{~ns}$ long. Such a long recovery 


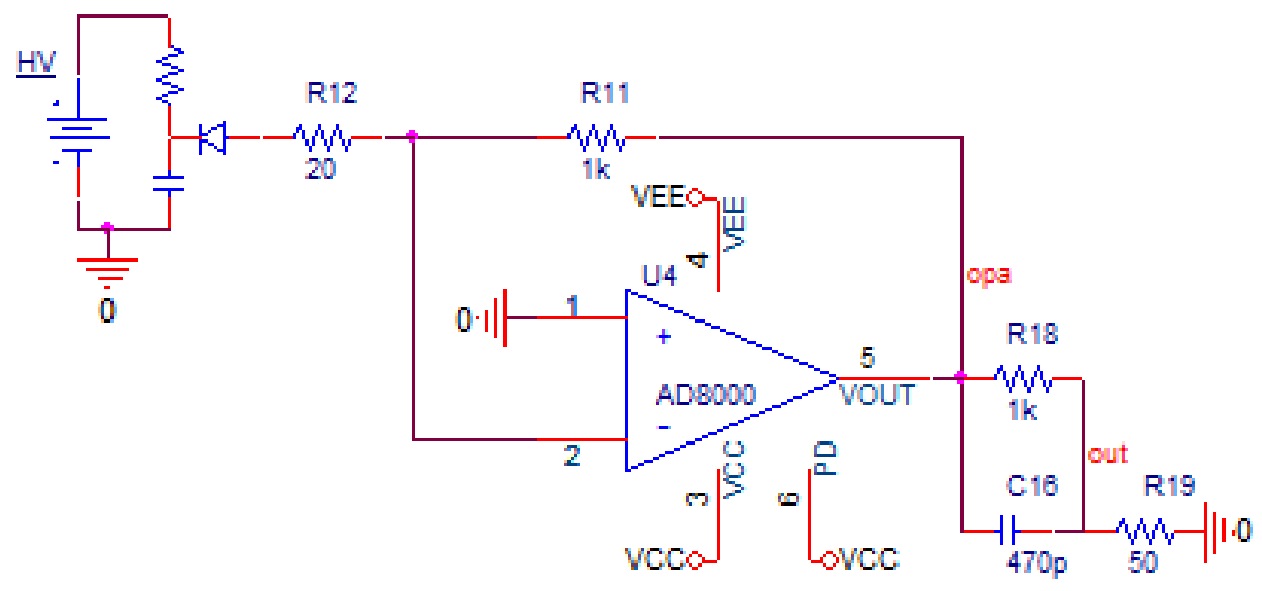

Figure 2: Schematic view of the SiPM and the AD8000 OPA. The Pole-Zero cancellation circuit is also drawn.

time can be problematic in high-background environments, like the one expected for the CTA experiment. For this purpose, a Pole-Zero cancellation circuit [3] was added to the preamplifier design (see Figure 2). A $470 \mathrm{pF}$ capacitance was placed in parallel to a $1 \mathrm{k} \Omega$ resistance and closed over a $50 \Omega$ load. The signal taken from this Pole-Zero cancellation network is shown in the right panel of Figure 3. The absence of the long tail is clearly visible, and the same amplitude is almost preserved.
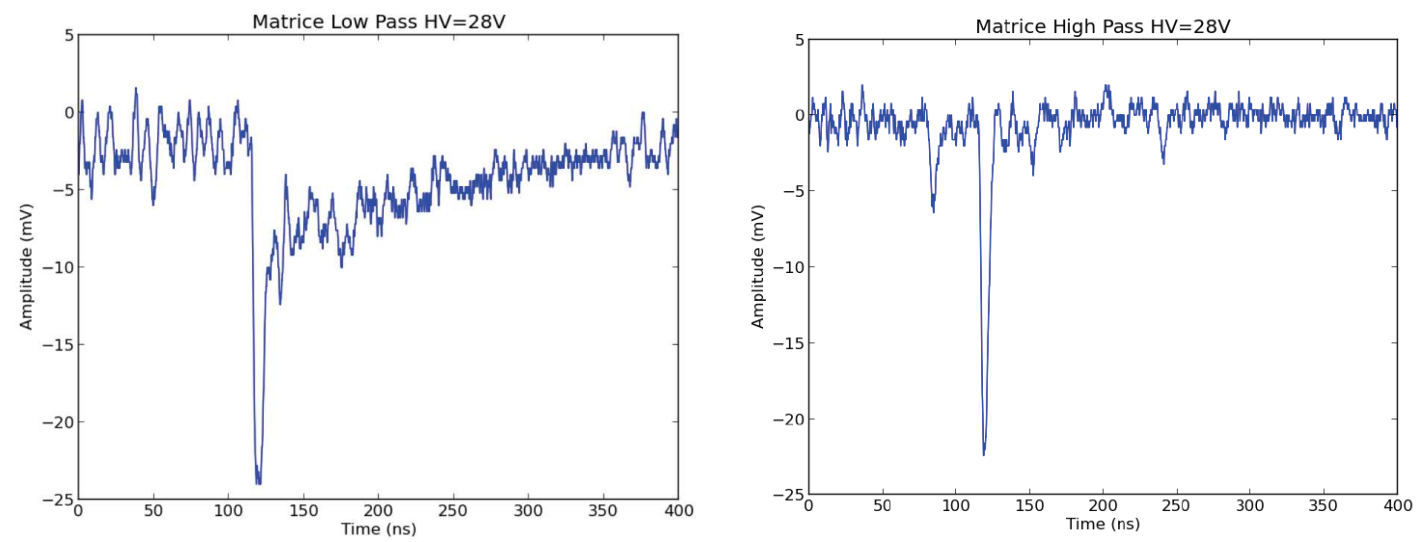

Figure 3: Current signals from the preamplifier obtained without (left panel) and with the Pole-Zero cancellation circuit (right panel).

\section{Prototype performance}

We tested the coupling of the SiPM matrix with the electronics by means of a pulsed-mode laser light. Four out of the 16 signals were sent to a TDS5104B oscilloscope, with a signal sampling of $5 \mathrm{GHz}$. Two screen-shots are shown in Figure 4. The left panel refers to the dark count signals. 

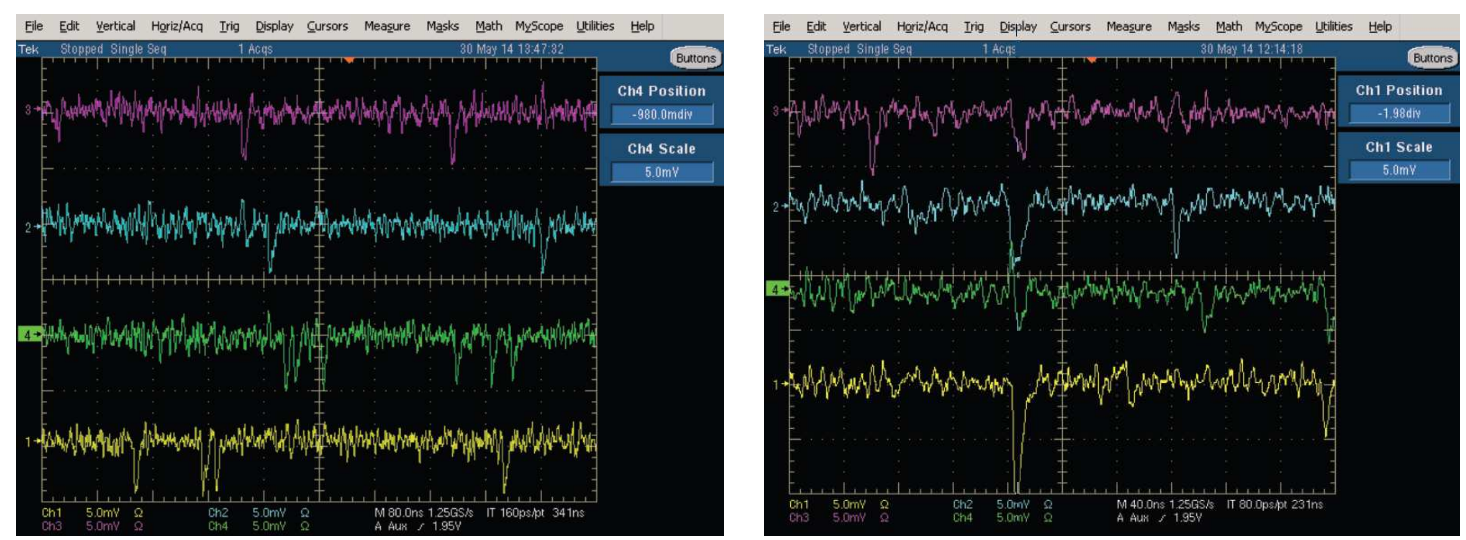

Figure 4: Screenshots from the oscilloscope's monitor. Left panel: Signals sampled in the dark; Right panel: Signals sampled in coincidence with the laser pulse.

Here, the average amplitude per single p.e. is about $5 \mathrm{mV}$ and the average distance time is about $200 \mathrm{~ns}$, corresponding to a total dark rate of about $500 \mathrm{kHz} / \mathrm{mm}^{2}$. It is worth noting that the SiPMs adopted for this analysis represent a very preliminary production by FBK. More recently developed sensors show an improvement in the dark count rate by an order of magnitude. The right panel of Figure 4 shows the signal sampling in the case of a laser event, characterized by the four SiPM signals being coincident in time.

We studied the amplitude of the signal from a single SiPM by covering all other SiPMs. An example amplitude spectrum is shown in the top left panel of Figure 5. The amplitude distribution of all p.e. events clearly exhibits a Poissonian shape, as can be seen top right panel of Figure 5. We also studied the linearity of the SiPM electronics chain. The bottom left panel of Figure 5 displays a linear fit, which indicates a good linear correlation of the signal amplitude vs. the number of converted photo-electrons. Finally we evaluated the signal-to-noise ratio (SNR) by calculating the ratio between the gain (i.e. the position of the single p.e. amplitude) to the width of the electronic noise (see the bottom right panel of Figure 5).

In summary, we conclude that our device shows a gain of $5.6 \mathrm{mV} / \mathrm{p}$.e. with a SNR of about 8.3 for an $\mathrm{OV}$ of roughly $2 \mathrm{~V}$.

\section{Future Work}

INFN is currently producing a cluster of 7 or more $3 \times 3$ (or $6 \times 6$ ) SiPMs of 1 inch diameter, which can be suitable for telescopes with big cameras, like CTA's large size telescopes (LSTs). The left panel of Figure 6 shows a preliminary design of a PCB for housing a matrix of 16 SiPMs and an electronics optimized to collect the signals of all these sensors and to fed out the signal of only one pixel. The coupling of this high dimensions pixel with a light guide (shown in the right panel) is also being exploited. The first prototypes have been produced with a cooperation between INFN Padova and INFN Perugia and will be tested on the MAGIC telescope.

Moreover, FBK is continuously producing and upgrading devices with a reduced dark count rate, an increased PDE and an optimized geometry, in order to reduce the dead area zone. Further studies are in progress in order to optimize the current signal tail in order to limit the impact of the 

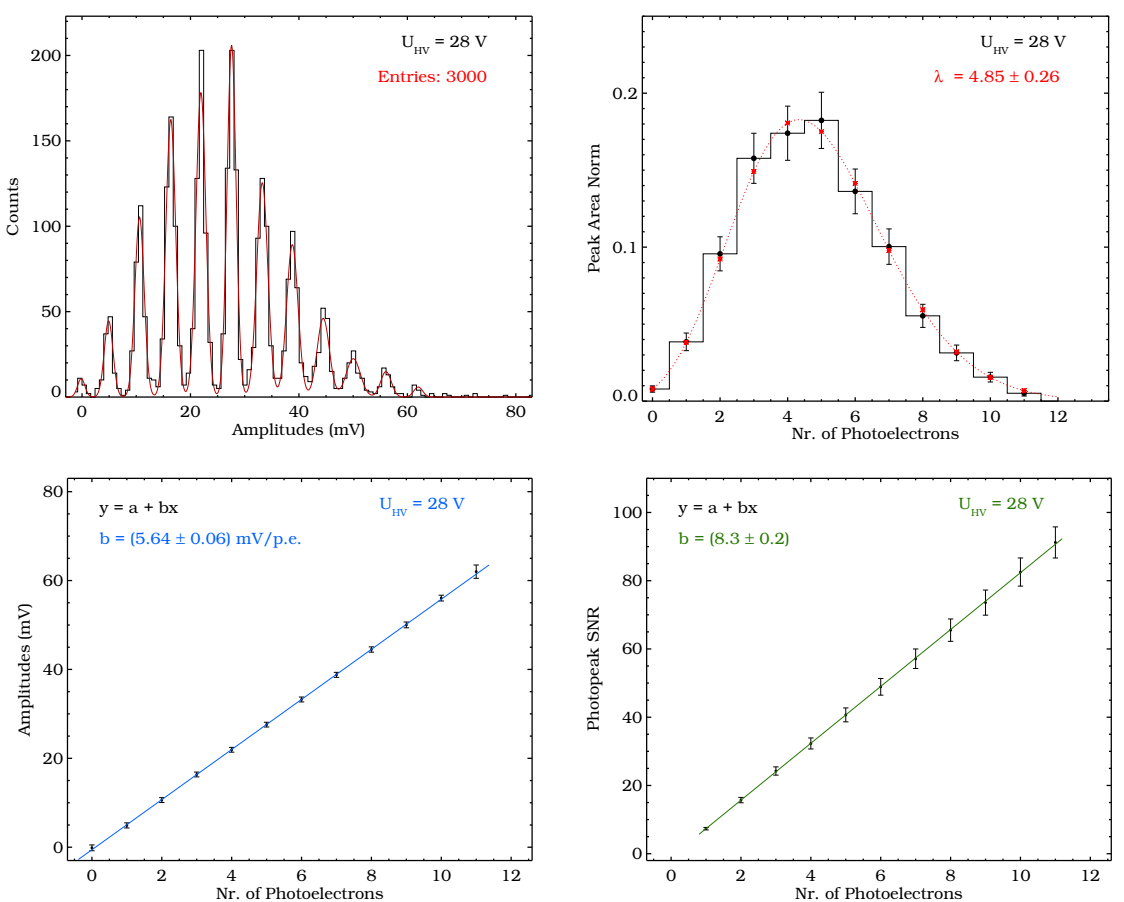

Figure 5: Top left panel: Amplitude spectrum from a single SiPM. Top right panel: Amplitude distribution. Bottom left panel: Gain. Bottom right panel: SNR.

diffuse light background to the trigger efficiency and signal reconstruction. For this purpose, more tests are currently under investigation.
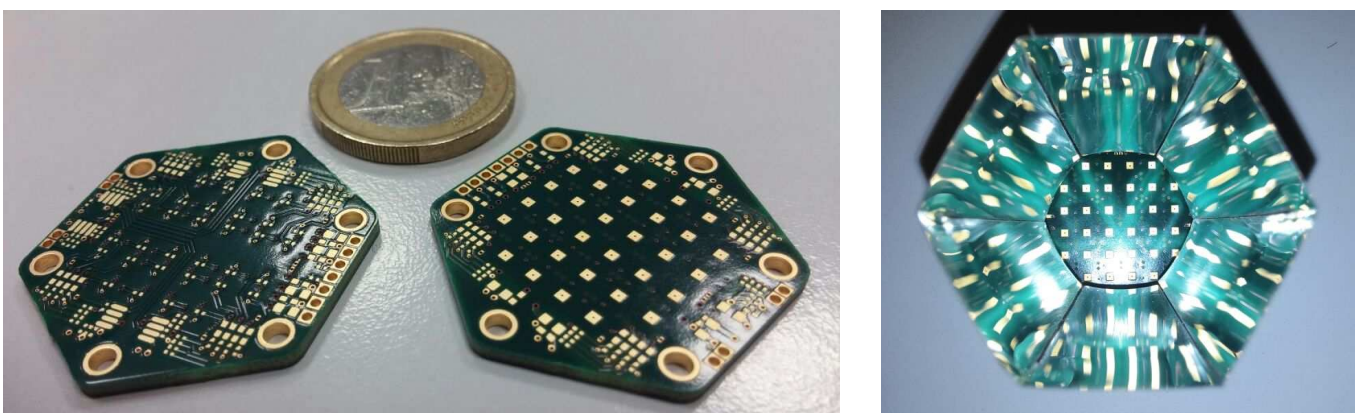

Figure 6: High dimensions pixel: Left panel: array of SiPMs and electronics; Right panel: the SiPM array seen through the light guide.

\section{References}

[1] http://www.fbk.eu/

[2] Pro, T., et al., 2013, "New Developments of Near-UV SiPMs at FBK", IEEE TRANSACTIONS ON NUCLEAR SCIENCE, Volume: 60, Issue:3, Part:3

[3] Gola, A., Piemonte, C., Tarolli A., 2013, "Analog Circuit for Timing Measurements With Large Area SiPMs Coupled to LYSO Crystals", IEEE TRANSACTIONS ON NUCLEAR SCIENCE, Volume: 60, Issue:2, Part:2 